\title{
Preoperative very short-term, high-dose erythropoietin administration diminishes blood transfusion rate in off-pump coronary artery bypass: A randomized blind controlled study
}

Luca Weltert, MD, Stefano D’Alessandro, MD, Saverio Nardella, MD, Fabiana Girola, MD, Alessandro Bellisario, MD, Daniele Maselli, MD, and Ruggero De Paulis, MD

Objective: Human recombinant erythropoietin has been used to obtain a rapid increase in red blood cells before surgery. Previously, the shortest preparatory interval has been 4 days, but at the European Hospital only 2.4 days on average separate hospitalization and surgery. We therefore proposed a randomized blind trial to test the efficacy of high-dose erythropoietin for very short-term administration.

Methods: All patients presenting with a diagnosis of isolated coronary vessel disease were randomized to either erythropoietin therapy or a control group. Patients with a creatinine level greater than $2 \mathrm{mg} / \mathrm{dL}$ or hemoglobin level greater than $14.5 \mathrm{~g} / \mathrm{dL}$ were excluded. Hemoglobin values were collected preoperatively and on postoperative days 1 and 4 . Blood loss and blood transfusion rate were recorded at the time of discharge.

Results: We enrolled 320 consecutive patients in the study. No significant difference was found in preoperative parameters, postoperative blood loss, or mean preoperative hemoglobin levels. On postoperative day 4 , mean hemoglobin was $15.5 \%$ higher in the erythropoietin group $(10.70 \pm 0.72 \mathrm{~g} / \mathrm{dL}$ vs $9.26 \pm 0.71 \mathrm{~g} / \mathrm{dL} ; P<.05)$. This group required 0.33 units of blood per patient, whereas the controls required 0.76 units per patient (risk ratio 0.43 , $P=.008)$.

Conclusion: A significant reduction in transfusion rate and a significant increase in hemoglobin values were observed in the erythropoietin group. No adverse events related to erythropoietin administration were recorded. A very short preoperative erythropoietin administration seems to be a safe and easy method to reduce the need for blood transfusions. (J Thorac Cardiovasc Surg 2010;139:621-7)

Earn CME credits at

http://cme.ctsnetjournals.org

The use of blood conservation techniques is important in cardiac surgery, because postoperative bleeding is common and allogenic blood transfusion carries the risk of transfusion reactions and infection. Specifically, in isolated coronary artery bypass grafting (CABG) the transfusion of allogenic blood increases the risk for postoperative atrial fibrillation, worsens health-related quality of life, and reduces long-term survival. ${ }^{1-3}$ According to recent reports, more than one third of patients undergoing elective $\mathrm{CABG}$ still require allogenic blood, and approximately $20 \%$ of transfusions are associated with cardiac surgery. Erythropoietin with and without preoperative autologous blood donation is one way to minimize allogenic transfusion. ${ }^{4}$ Erythropoietin is a 165 amino acid glycoprotein hormone with a molecular weight of approximately $30 \mathrm{kDa}$. It is synthesized

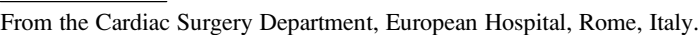
Disclosures: None.

Received for publication April 30, 2009; revisions received Sept 9, 2009; accepted for publication Oct 4, 2009; available ahead of print Dec 30, 2009.

Address for reprints: Luca Weltert, MD, Cardiac Surgery Department, European Hospital, Rome, Italy (E-mail: lweltert@gmail.com).

$0022-5223 / \$ 36.00$

Copyright (c) 2010 by The American Association for Thoracic Surgery doi:10.1016/j.jtcvs.2009.10.012
}

primarily by the kidneys in adults and by the kidneys and liver in the fetus. The ratio between kidney and liver erythropoietin in adults is 9:1. Its primary role involves the prevention of programmed cell death (apoptosis) of erythrocyte precursors. Erythropoietin induces erythropoiesis by promoting the proliferation and differentiation of erythroid progenitor cells, the main target cell being the colony-forming unit erythroid.

In addition to erythropoietin's well-known effect on red blood cell mass in response to changes in tissue oxygenation, many investigations have shown that it also exerts a protective role against tissue ischemia. It is believed that this is achieved both directly by activating multiple biochemical mechanisms that provide anti-apoptotic, anti-oxidative, and anti-inflammatory responses to hypoxia/anoxia, and indirectly via its angiogenic potential by inducing a systematic oxygen supply to the ischemic tissue. ${ }^{5}$

Recombinant human erythropoietin (HRE) was developed in the mid-1980s and is commercially available in several forms. Erythropoietin combined with oral iron therapy is used to treat anemia (hemoglobin $[\mathrm{Hb}]<13 \mathrm{~g} / \mathrm{dL}$ ) in renal failure, associated with chemotherapy or human immunodeficiency virus, and when given preoperatively, to reduce transfusion in a wide range of operations. Abundant evidence, including 4 meta-analyses, ${ }^{6-9}$ exists to justify the preoperative administration of HRE to reduce preoperative anemia, especially in patients undergoing autologous blood donation ${ }^{10-13}$ and in children. ${ }^{14,15}$ Erythropoietin seems to 


\section{Abbreviations and Acronyms \\ ANOVA $=$ analysis of variance \\ $\mathrm{CABG}=$ coronary artery bypass grafting \\ $\mathrm{Hb}=$ hemoglobin \\ HRE = human erythropoietin}

be safe and effective for the improvement of preoperative anemia. Preoperative interventions using HRE seem justified for elective patients with diminished blood volume because of the high risk of excessive blood transfusion in this subset. Still fewer objective data are available regarding the use of HRE to treat peri- and postoperative anemia. Because the onset of drug action is 4 to 6 days, it has been considered necessary to administer HRE a few days before the operation. There are some conditions in which the production of endogenous HRE is limited: beta-blocker therapy, cytokines stimulated by the inflammatory response associated with cardiopulmonary bypass, and perioperative renal ischemia. Likewise, careful perioperative management may improve tissue oxygen delivery and suppress endogenous HRE production despite postoperative anemia. All of these factors support the addition of preoperative (ie, a few days before surgery) administration of HRE to treat reduced red blood cell volume in selected patients. ${ }^{16,17}$ So far, the shortest preparatory administration interval has been up to 4 days preoperatively.

At the European Hospital, an average of only 2.4 days separate hospitalization and surgery, and inpatient optimization procedures are pushing this limit even further. This situation is common in many high-volume centers, making any protocol requiring the patient to wait more than 3 days before operation unattractive and practically useless. We therefore proposed a randomized blind trial to test the efficacy of high-dose HRE in very short-term administration, a protocol that does not change the usual interval between admission and surgery. Should the advantages of HRE administration be present even with such a short interval, its use could be advisable as part of the routine strategy to minimize the use of allogenic blood transfusions.

\section{MATERIALS AND METHODS}

All patients presenting with a diagnosis of isolated coronary vessel disease at the European Hospital were considered for the study. Exclusion criteria were the presence of high $\mathrm{Hb}$ values $(>14.5 \mathrm{~g} / \mathrm{dL}$, or hematocrit $>$ $44 \%$ ), confirmed renal impairment (creatinine $>2 \mathrm{mg} / \mathrm{dL}$ ), or the need for on-pump revascularization. The decision to exclude the latter was made to avoid the confounding factor of extracorporeal circulation, which implies an increase in hemodilution and blood loss.

All patients' risk factors for ischemic heart disease (family history, the presence of diabetes, hypercholesterolemia, smoking, obesity, hypertension); factors included in the EuroSCORE analysis (age, gender, chronic pulmonary obstructive disease, the presence of extracardiac arteriopathy, neurologic dysfunction, previous cardiac surgery, serum creatinine, active endocarditis, critical preoperative state, unstable angina, left ventricle dysfunction, recent myocardial infarct, pulmonary hypertension, emergency conditions, postinfarct septal rupture); and biometric parameters (height, weight, and body surface area) were collected and stored in the database before the results of randomization were known. A custom simple application running in Windows XP (Microsoft Corp, Redmond, WA) was used to obtain randomization tables, and the next value of the table was kept secret until a suitable patient was enrolled. This study adheres to the CONSORT principles, and patients followed the CONSORT 2005 FlowChart.

Informed consent was then obtained, and all aspects of the operation, drug administration, and any other relevant matter were explained to the patient by one of the investigators. The study followed the Helsinki Declaration requirements for randomized case-control trials and was approved by the institutional review board of the European Hospital.

The patients randomized to the HRE group received 14,000 IU via subcutaneous administration 2 days before the operation, 14,000 IU on the next day, $8000 \mathrm{IU}$ on the morning of the operation, $8000 \mathrm{IU} 1$ day after operation, and $8000 \mathrm{IU}$ on postoperative day 2 . The control group received no treatment. Despite known interactions of HRE with other medications (eg, beta-blockers), all standard therapies were maintained until the day of operation. The patients, nurses, and ward physician knew whether HRE was being administered or not, but the investigators did not, nor did they have any chance to influence the clinical decision as to whether or not to give allogenic blood, thus fulfilling the conditions for a single-blinded study.

The primary end point was the need for allogenic transfusion. The secondary end point was the $\mathrm{Hb}$ value on postoperative day 4 . Ancillary objectives were the $\mathrm{Hb}$ trend from baseline to day 4 with and without HRE administration, and the cost-effectiveness of HRE versus the reduced need for transfusion.

Transfusion need was triggered by $\mathrm{Hb}$ levels less than $8.0 \mathrm{~g} / \mathrm{dL}$, and the same criteria were applied to both groups. Hb values were automatically collected by the central laboratory computer on the day of admission, on the day of surgery, and on postoperative day 4 . The number of units transfused, the amount of blood loss, any kind of adverse reaction, and the patient's outcome were collected directly from the clinical records 2 weeks after discharge. All patients underwent outpatient follow-up approximately 45 days after surgery, at which time all adverse events were investigated.

All data were processed by SPSS version 16 (SPSS Inc, Chicago, Ill), including sample sizing, analysis of variance (ANOVA), and risk analysis. A preliminary power estimation analysis suggested that 160 patients per sample were needed to obtain a $90 \%$ power goal, considering an alpha error level of $5 \%$ and expecting the incidence of transfusion to decrease from the previously observed $30 \%$ to $15 \%$.

The first step of analysis was to ascertain whether the randomization process had been effective in controlling as many known confounding factors as possible. ANOVA of the dependent variables "HRE or control" was performed (ANOVA and univariate ANOVA as needed, 95\% confidence interval, $P$.05; full list of parameters and $P$ values are shown in Table 1) on all preoperative data, namely, general cardiovascular risk parameters, EuroSCORE risk parameters, biometric parameters, and blood loss.

The second step was to verify whether the 2 groups really differed in terms of $\mathrm{Hb}$ value on day 4, transfusion rate, relative risk of undergoing allogenic blood transfusion, duration of hospital stay, and adverse events. Every comparison was tested with several statistical techniques (Student $t$ test, univariate regression, Yates' continuity-corrected chi-square test as needed) as appropriate.

The general statistical aspect of the study was kept as simple as possible in the belief that even if sophisticated statistical techniques exist to control the influence of confounding factors, accurate planning remains the best tool.

\section{RESULTS}

Recruitment took place between October 1, 2007, and September 31, 2008, and 400 patients were assessed for 
eligibility; 80 patients were excluded from the study (60 patients did not meet the inclusion criteria, 11 patients refused to participate, and 9 patients were excluded for other reasons). Of the remaining 320 patients, 158 were allocated to HRE administration and all received the intended treatment; 162 were allocated to the control group. Two patients in the HRE group and 1 patient in the control group were converted to on-pump revascularization. These patients were considered as intention-to-treat and were therefore included in the analysis.

ANOVA confirmed the efficacy of the randomization process, because all variables analyzed proved to have no statistically significant difference between the 2 groups (Table 1). The baseline demographic and clinical characteristics of each group are also summarized in Table 1. Both groups used the cell-saver system during the operation and received standardized doses of a fibrinolytic agent (tranexamic acid). There was no statistical difference between the groups in terms of assignment to a specific investigator $(P=.323)$. For the HRE and control groups, the time in the operating suite was 168 and 146 minutes, respectively $(P=.342)$; the number of grafts performed was 3.11 and 3.16 , respectively $(P=.677)$; the percentage of take-backs for bleeding was $1.30 \%$ and $1.25 \%$, respectively $(P=$ .32 ); and the clotting factors transfused were 0.08 and 0.09 units of plasma per patient, respectively $(P=.612)$, and 0.04 and 0.03 units of platelets per patient, respectively $(P=.132)$. Baseline $\mathrm{Hb}$ showed no significant difference between the groups $(P>.3)$, nor did blood loss (587 vs $616 \mathrm{~mL}, P=.43$ ).

On postoperative day $4, \mathrm{Hb}$ was $10.70 \pm 0.72 \mathrm{~g} / \mathrm{dL}$ in the HRE group and $9.26 \pm 0.71 \mathrm{~g} / \mathrm{dL}$ in the control group $(P=$ $.03,95 \%$ confidence interval). The HRE group required 0.32 units of blood per patient, whereas the control group required 0.76 units per patient, and this difference proved to be highly significant $(P=.008)$. In greater detail, $15.82 \%$ of patients $(25 / 158)$ in the HRE group required transfusion, $8.86 \%$ (14) requiring 1 unit, $4.43 \%$ (7) requiring 2 units, and $2.53 \%$ (4) requiring 3 or more units. In the control group, $37.2 \%$ of patients (60/162) required transfusion, $11.72 \%$ (19) requiring 1 unit, $19.75 \%$ (32) requiring 2 units, and $5.55 \%$ (9) requiring 3 or more units. The risk ratio of being exposed to allogenic blood was $0.425(P=.007$, $95 \%$ confidence interval) for the HRE group versus the control group (Figure 1).

Table 2 shows the adverse events analysis, with differences in terms of 45-day mortality, in-hospital complications, and mid-term morbidity. Briefly, there was no difference in 45-day mortality (3 patients in each group, $1.92 \%$ vs $1.86 \% P=.672$ ). In regard to in-hospital complications, patients in the control group had a lower incidence of cardiac tamponade and increased plasma liver enzymes, a higher incidence of positive blood, sputum, and urine cultures, and higher peak troponin values. On mid-term follow-
TABLE 1. Baseline characteristics of the study population and analysis of variance $P$ value

\begin{tabular}{|c|c|c|c|}
\hline & HRE & Control & $P$ alue \\
\hline Family history & $40.6 \%$ & $38.2 \%$ & .342 \\
\hline Obesity & $19.8 \%$ & $19.6 \%$ & .98 \\
\hline Diabetes & $44.4 \%$ & $40.5 \%$ & .342 \\
\hline Hypercholesterolemia & $59.3 \%$ & $67.7 \%$ & .851 \\
\hline Smoking habit & $52.1 \%$ & $53.3 \%$ & .349 \\
\hline Hypertension & $77.8 \%$ & $78.5 \%$ & .260 \\
\hline EuroSCORE & $7.94 \pm 8.07$ & $6.82 \pm 10.39$ & .372 \\
\hline Age & $66.9 \pm 9.11$ & $66.4 \pm 10.8$ & .735 \\
\hline Gender & Male, $84 \%$ & Male, $83 \%$ & .169 \\
\hline $\begin{array}{l}\text { Chronic pulmonary } \\
\text { obstructie disease }\end{array}$ & $32.1 \%$ & $16.5 \%$ & .079 \\
\hline $\begin{array}{l}\text { Presence of extracardiac } \\
\text { arteriopathy }\end{array}$ & $23.5 \%$ & $15.8 \%$ & .454 \\
\hline $\begin{array}{l}\text { Neurologic dysfunction } \\
\text { disease }\end{array}$ & $2.9 \%$ & $1.9 \%$ & .913 \\
\hline Preious cardiac surgery & $1.8 \%$ & $0.6 \%$ & .332 \\
\hline Serum creatinine & $1.08 \pm 0.30$ & $1.14 \pm 0.32$ & .206 \\
\hline Seere renal impairment & $0 \%$ & $0 \%$ & NA \\
\hline Presence of actie endocarditis & $0 \%$ & $0 \%$ & NA \\
\hline Critical preoperatie state & $0 \%$ & $0.6 \%$ & NA \\
\hline Unstable angina & $27.9 \%$ & $26.6 \%$ & .177 \\
\hline Ejection fraction & $54.11 \pm 7.87$ & $53.03 \pm 8.82$ & .344 \\
\hline Recent myocardial infarct & $34.4 \%$ & $30.4 \%$ & .344 \\
\hline Pulmonary hypertension & $0 \%$ & $0.6 \%$ & .487 \\
\hline Postinfarct septal rupture & $0 \%$ & $0 \%$ & NA \\
\hline Height & $169 \pm 7.8$ & $168 \pm 8.5$ & .426 \\
\hline Weight & $80.94 \pm 13$ & $76.83 \pm 11.41$ & .344 \\
\hline Body surface area $\left(\mathrm{m}^{2}\right)$ & $1.74 \pm 0.88$ & $1.67 \pm 0.77$ & .227 \\
\hline Baseline $\mathrm{Hb}(\mathrm{g} / \mathrm{dL})$ & $13.18 \pm 1.21$ & $13.44 \pm 1.20$ & .183 \\
\hline Blood loss (mL) & $605 \pm 373$ & $554 \pm 326$ & .479 \\
\hline
\end{tabular}

$H b$, Hemoglobin; $H R E$, human erythropoietin; $N A$, not applicable.

up (45 days), the control group showed a higher incidence of neurologic events and deep vein thrombosis. All differences presented with a low level of significance. The 2 populations did not differ for the other parameters considered.

With $€ 243$ as the protocol expense for the HRE group, the cost of 1 unit of blood being $€ 270$, the saving of approximately half a unit of blood per patient was not cost-effective. Nevertheless, a net reduction of 1 unit of blood for every 2 patients is an acceptable gain in terms of overall risk reduction. Moreover, by focusing on a higher-risk population, starting with baseline $\mathrm{Hb}$ less than $10.5 \mathrm{~g} / \mathrm{dL}$, the advantage in terms of spared blood units is higher ( 0.28 units/ patient vs 0.99 units/patient, $P=.012$ ).

There was a slight difference in the duration of hospital stay, with an advantage for the HRE group of 5.32 days versus 5.89 days, but the difference was not significant $(P=$ .08). However, if confirmed, the increased length of stay of 0.57 days per patient would increase the cost of the control group by $€ 456$ per patient, thus making the protocol eventually convenient. 


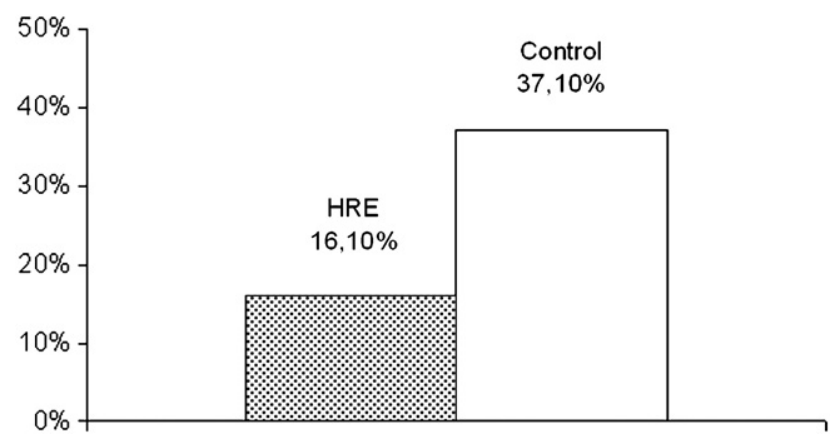

FIGURE 1. Risk of exposure to allogenic blood transfusion $(P=.007)$. $H R E$, Human erythropoietin.

\section{DISCUSSION}

The focus of this randomized blind study was to assess whether a high dose of HRE administered shortly before surgery is advantageous, safe, and cost-effective in reducing the risk of exposure to allogenic blood transfusion.

The importance of blood conservation techniques is well known in cardiac surgery, because postoperative bleeding is common and allogenic transfusion carries the risks of both transfusion reactions and infection transmission. Despite all efforts, approximately one third of patients undergoing elective CABG still require allogenic blood.

The guidelines for blood conservation have emphasized the need for a multifactorial approach, and erythropoietin administration with and without preoperative autologous blood donation is one of the recommended modalities to minimize allogenic blood transfusion.

Although recombinant human HRE was developed in the mid-1980s, and abundant evidence, including 4 meta-analyses, ${ }^{6-9}$ exists to justify its preoperative administration to reduce perioperative anemia, there is still no consensus regarding its use. One main practical concern is that obtaining the full effect of HRE takes more time than is usually available in a high-volume, efficiency-oriented center. A typical preoperative HRE regimen is costly, and there is uncertainty about its cost-effectiveness for patients undergoing autologous blood donation before cardiac procedures. Evidence supporting the preoperative use of HRE in anemic patients $(\mathrm{Hb}<13 \mathrm{~g} / \mathrm{dL})$ without autologous pre-donation is less compelling, but still supportive. Most of the literature supporting the use of HRE to reduce preoperative anemia is anecdotal and relates to successful case reports in a handful of patients, especially Jehovah's Witnesses. Because preoperative anemia increases mortality and morbidity in cardiac procedures, HRE can be expected to reduce it by augmenting red cell mass in anemic patients treated with iron, if given more than 1 week before operation. This recommendation is based on limited evidence and logical consensus.

In our search for a strategy suitable for routine use, we thought that a boost to red cell growth could only be convenient if present shortly after HRE administration, thereby preventing the patient waiting longer than strictly necessary for the operation. To our knowledge, no other published studies had ever tested the efficacy of this drug in such a context.

Many studies based on decision analysis models concluded that the use of HRE reduces the risk of exposure to allogenic blood transfusion. ${ }^{18,19}$ Our experience shows that HRE administration has a significantly $(P=.007)$ protective effect, roughly halving this risk, and significantly $(P$ $=.03$ ) increases the $\mathrm{Hb}$ value on day 4 after surgery by $15.55 \%$.

The safety of HRE is an important area to address. D'Ambra and colleagues ${ }^{20}$ reported an increased frequency of mortality during the study or within 2 months of discontinuation of the double-blind therapy, but their results were not significant $(P=.06)$. However, this was the only study in which HRE administration showed an increase in mortality, albeit not significantly. Hayashi and colleagues ${ }^{21}$ reported that 2 patients were removed from their trial because of the adverse events of HRE therapy (fatigue and dizziness developed in 1 patient, and skin rash and hypertension developed in 1 patient). Although hypertension is more likely to be associated with long-term HRE therapy, there have been reports of hypertension developing during short-term treatment in individual patients without a history of hypertension. ${ }^{22}$ Moreover, D'Ambra and colleagues found no significant difference in the nonfatal complications of therapy between the HRE and the placebo groups.

We observed no difference in terms of mortality 45 days after the operation. The analysis of adverse events occurring during the hospitalization seems to indicate that HRE administration is not an increased risk factor. The analysis of adverse events occurring in the first 45 days after operation showed no difference in terms of myocardial infarction, renal failure, pneumonia, or wound infection. However, the control group had a slightly higher incidence of neurologic ischemic events and deep vein thrombosis. No new-onset hypertension was observed in either group.

Our data show that short-term, high-dose administration of HRE does not correlate with increased complications and should therefore be considered safe. One possible explanation for this is the structure of the protocol itself. We know that increasing blood viscosity is a prothrombotic procedure. This has always been the major concern regarding HRE use in patients with vascular disease. However, in this specific case patients at risk of developing high hematocrit $(\mathrm{Hb}$ $>14.5$ ) are excluded from the protocol. Moreover, 48 hours after initial administration of the drug another event occurs to dramatically reduce the risk of thrombosis, that is, periand postoperative blood loss. Bleeding occurs just before the peak effect of HRE, thereby minimizing the risk of excessive blood viscosity; in fact, no patient had an $\mathrm{Hb}$ level greater than $14.0 \mathrm{~g} / \mathrm{dL}$ on the day of discharge.

Finally, uncertainty exists about the cost-effectiveness of HRE for patients undergoing cardiac procedures. A typical 
TABLE 2. Postoperative morbidity analysis

\begin{tabular}{|c|c|c|c|}
\hline & HRE & Control & $P$ value \\
\hline ICU stay $(\mathrm{d})$ & 1.571 & 1.567 & .89 \\
\hline Perioperative MI (\%) & 2.02 & 2.31 & .53 \\
\hline Peak troponin level (nmol/L) & 0.841667 & 4.576694 & .05 \\
\hline Epileptic syndrome $(\%)$ & 0.00 & 0.63 & .12 \\
\hline Focal neurologic damage $(\%)$ & 0.00 & 0.63 & .14 \\
\hline Generalized neurologic damage $(\%)$ & 0.00 & 0.63 & .16 \\
\hline Total bleeding $(\mathrm{mL})$ & 587.1429 & 616.0484 & .43 \\
\hline Bleeding at $6 \mathrm{~h}(\mathrm{~mL})$ & 198.5185 & 271.2941 & .28 \\
\hline Bleeding at $12 \mathrm{~h}(\mathrm{~mL})$ & 299.2593 & 360.5882 & .42 \\
\hline Bleeding at $24 \mathrm{~h}(\mathrm{~mL})$ & 375.6 & 452.8 & .36 \\
\hline Revision for hemostasis (\%) & 1.30 & 1.25 & .32 \\
\hline Cardiac tamponade $(\%)$ & 2.00 & 0.63 & .05 \\
\hline Atrial fibrillation $(\%)$ & $2.5 \%$ & $2.4 \%$ & .45 \\
\hline Patients transfused in ICU $(\%)$ & 22.00 & 36.25 & .001 \\
\hline Acute respiratory distress syndrome $(\%)$ & 4.00 & 4.32 & .43 \\
\hline Acute pulmonary edema $(\%)$ & 0.00 & 0.00 & NA \\
\hline Pneumonia $(\%)$ & 0.00 & 0.00 & NA \\
\hline Need for reintubation $(\%)$ & 0.00 & 0.63 & .22 \\
\hline Increase in liver enzymes $(\%)$ & 2.00 & 0.00 & .03 \\
\hline Increase in pancreatic enzymes $(\%)$ & 0.00 & 0.00 & na \\
\hline Bowel ischemia $(\%)$ & 0.00 & 0.00 & na \\
\hline Creatinine level before surgery $(\mathrm{mmol} / \mathrm{L})$ & 1.1825 & 1.104889 & .34 \\
\hline Maximum creatinine level $(\mathrm{mmol} / \mathrm{L})$ & 1.189744 & 1.074715 & .23 \\
\hline First 24-h urine output (mL) & 1925.897 & 1891.009 & .45 \\
\hline Use of fenoldopam $(\%)$ & 8.00 & 5.00 & .07 \\
\hline Need for CVVHDF & 2.00 & 0.63 & .09 \\
\hline Need for insulin infusion $(\%)$ & 18.00 & 15.00 & .1 \\
\hline Positive blood culture $(\%)$ & 0.00 & 0.63 & .02 \\
\hline Positive urine culture $(\%)$ & 0.00 & 0.63 & .03 \\
\hline Positive sputum culture ( $\%)$ & 0.00 & 0.63 & .02 \\
\hline Positive sternal wound culture (\%) & 0.00 & 0.00 & NA \\
\hline Fluid balance on discharge from ICU $(\mathrm{mL})$ & 503.6923 & 447.9344 & .32 \\
\hline Central venous pressure $(\mathrm{mm} \mathrm{Hg})$ & 8.297297 & 8.237705 & .88 \\
\hline Length of stay after operation (d) & 5.52 & 5.89 & .065 \\
\hline Neurologic complications at $45 \mathrm{~d}(\%)$ & 0.00 & 1.88 & .02 \\
\hline Long-term wound infection $(\%)$ & 2.00 & 1.88 & .65 \\
\hline Deep vein thrombosis $(\%)$ & 0.00 & 0.63 & .03 \\
\hline Acute hypertension onset $(\%)$ & 0.00 & 0.00 & NA \\
\hline Renal failure $(\%)$ & 0.00 & 0.00 & NA \\
\hline 45-d mortality $(\%)$ & 1.92 & 1.86 & .67 \\
\hline
\end{tabular}

$C V V H D F$, Continuous veno-venous hemodiafiltration; $H R E$, human erythropoietin; $I C U$, intensive care unit; $M I$, myocardial infarction; $N A$, not applicable.

preoperative regimen of HRE is costly, and at least 2 studies showed that it was not cost-effective. ${ }^{18,19}$ We carried out an ancillary analysis considering the cost-effectiveness of the procedure: Our protocol expense was $€ 243$, and 1 unit of blood requested electively cost $€ 270$, which increased to $€ 390$ euros if requested in an emergency. The use of HRE resulted in a saving of 1 unit of blood for every 2 patients, and therefore was not cost-effective, even though the patient had a net gain in terms of risk reduction for immunologic reaction, infective disease transmission, and atrial fibrillation, as well as a better health-related quality of life after cardiac surgery and even long-term survival after CABG. ${ }^{1-3}$ Strata analysis showed that the protective effect is far greater for patients with baseline $\mathrm{Hb}$ less than $10.5 \mathrm{~g} / \mathrm{dL}$; therefore, $10.5 \mathrm{~g} / \mathrm{dL}$ can be considered a threshold to gain the maximum clinical benefit and minimize the added cost.

\section{STUDY LIMITATIONS}

There are some limitations to this study. First, the choice to exclude patients receiving on-pump surgery eliminated the bias related to extracorporeal circulation, but at the same time excluded from the study those patients with low red blood cell volume requiring urgent, high-risk surgery using cardiopulmonary bypass. Although these patients were 
a minority (in our population, planned on-pump revascularization was limited to 56 of 400 patients, $13.5 \%$ of the total), they were the patients who would have benefited more from the protocol. Second, the lack of long-term follow-up could miss some late complications that were not evident at 45 days. Third, possible interactions with other drugs (eg, beta-blockers) were not investigated, and the effect of increased perioperative oxygenation that might reduce the stimulus for endogenous erythropoietin production, making exogenous erythropoietin a more desirable option, was not taken into account. Finally, because of their lack of availability in Italy, neither aprotinin nor Amicar was used as part of the multifactorial blood-sparing strategy.

\section{CONCLUSIONS}

High-dose HRE administration, even in the very short term, leads to a significant reduction in transfusion rate and a significant increase in $\mathrm{Hb}$ values, thereby almost halving the risk of allogenic blood transfusion. No adverse events related to HRE administration were recorded. A very short preoperative administration of HRE seems to be a safe and easy method to reduce the need for allogenic blood transfusion and may be considered as part of a multifactorial strategy toward a further reduction for the need of transfusion in heart surgery.

\section{References}

1. Koch CG, Li L, Van Wagoner DR, Duncan AI, Gillinov AM, Blackstone EH. Red cell transfusion is associated with an increased risk for postoperative atrial fibrillation. Ann Thorac Surg. 2006;82:1747-56.

2. Koch CG, Khandwala F, Li L, Estafanous FG, Loop FD, Blackstone EH. Persistent effect of red cell transfusion on health-related quality of life after cardiac surgery. Ann Thorac Surg. 2006;82:13-20.

3. Koch CG, Li L, Duncan AI, et al. Transfusion in coronary artery bypass grafting is associated with reduced long-term survival. Ann Thorac Surg. 2006;81:1650-7.

4. Alghamdi A, Albanna M, Guru V, Brister S. Does the use of erythropoietin reduce the risk of exposure to allogeneic blood transfusion in cardiac surgery? A systematic review and meta-analysis. J Card Surg. 2006;21:320-6.

5. Paschos N, Lykissas M, Beris A. The role of erythropoietin as an inhibitor of tissue ischemia. Int J Biol Sci. 2008;4:161-8.

6. Monk TG. Preoperative recombinant human erythropoietin in anemic surgical patients. Crit Care. 2004;8(Suppl 2):45-8.

7. Goldberg MA. Perioperative epoetin alfa increases red blood cell mass and reduces exposure to transfusions: results of randomized clinical trials. Semin Hematol. 1997;34(Suppl 2):41-7.

8. YazicioGlu L, Eryilmaz S, Sirlak M, et al. Recombinant human erythropoietin administration in cardiac surgery. J Thorac Cardiovasc Surg. 2001;122:741-5.

9. Ascione R, Williams S, Lloyd C, Sundaramoorthi T, Pitsis A, Angelini G. Reduced postoperative blood loss and transfusion requirement after beating-heart coronary operations: a prospective randomized study. J Thorac Cardiovasc Surg. 2001;121:689-96.

10. Madi-Jebara SN, Sleilaty GS, Achouh PE, et al. Postoperative intravenous iron used alone or in combination with low-dose erythropoietin is not effective for correction of anemia after cardiac surgery. J Cardiothorac Vasc Anesth. 2004;18: 59-63.

11. Osaka M, Fukuda I, Ohuchi H. Aprotinin and recombinant human erythropoietin reduce the need for homologous blood transfusion in cardiac surgery. Jpn J Thorac Cardiovasc Surg. 1998;46:846-53.

12. Yoshikawa Y, Niwaya K, Hasegawa J, et al. Effect of blood conservation in openheart surgery: a comparison of 3 different methods. Kyobu Geka. 1994;47: 1059-62.

13. Price S, Pepper JR, Jaggar SI. Recombinant human erythropoietin use in a critically ill Jehovah's Witness after cardiac surgery. Anesth Analg. 2005;101:325-7.
14. Sonzogni V, Crupi G, Poma R, et al. Erythropoietin therapy and preoperative autologous blood donation in children undergoing open heart surgery. BrJ Anaesth. 2001;87:429-34.

15. Shimpo H, Mizumoto T, Onoda K, Yuasa H, Yada I. Erythropoietin in pediatric cardiac surgery: clinical efficacy and effective dose. Chest. 1997;111:1565-70.

16. Fisher JW. Pharmacologic modulation of erythropoietin production. Ann Rev Pharmacol Toxicol. 1988;28:101-22.

17. Means RT Jr. Clinical application of recombinant erythropoietin in the anemia of chronic disease. Hematol Oncol Clin North Am. 1994;8:933-44.

18. Coyle D, Lee KM, Fergusson DA, et al. Cost effectiveness of epoetin-alpha to augment reoperative autologous blood donation in elective cardiac surgery. Pharmacoeconomics. 2000;18:161-71.

19. Marchetti M, Barosi G. Cost-effectiveness of epoetin and autologous blood donation in reducing allogeneic blood transfusions in coronary artery bypass graft surgery. Transfusion. 2000;40:673-81.

20. D'Ambra MN, Gray RJ, Hillman R, et al. Effect of recombinant human erythropoietin on transfusion risk in coronary bypass patients. Ann Thorac Surg. 1997; 64:1686-93.

21. Hayashi J, Kumon K, Takanashi S, et al. Subcutaneous administration of recombinant human erythropoietin before cardiac surgery: a double-blind, multicenter trial in Japan. Transfusion. 1994;34:142-6.

22. Crosby E. Perioperative use of erythropoietin. Am J Ther. 2002;9:371-6.

\section{Discussion}

Dr Colleen Koch (Cleveland, Ohio). You present intriguing data demonstrating reduced red cell transfusion in patients receiving very short-term use of erythropoietin. I have 3 questions for you, and they pertain to the timing of administration, adverse events, and cost.

The first is with regard to timing. One of the biggest hurdles for the use of erythropoietin in the cardiac surgical setting is the short time interval between patient evaluation and the eventual operation. You demonstrated feasibility and effectiveness for reducing red cell transfusion as well as increasing day 4 hemoglobin. My question for you is, what were your indications for transfusion in this study? Meaning, was there a transfusion policy such that patients who were in your control group and erythropoietin group had a similar hemoglobin trigger that initiated a red cell transfusion?

Dr Weltert. Yes, of course we have a transfusion policy. We transfuse when the patient's hemoglobin decreases to less than 8 $\mathrm{g} / \mathrm{dL}$ in the absence of symptoms and when blood exsanguination as estimated by saturation of venous blood decreases to less than $50 \%$ even with optimal hemoglobin values, and, of course, this policy did not differ between the 2 groups.

Dr Koch. Wonderful. Now, my second question pertains to adverse events, and one of the major concerns with the use of erythropoietin in the cardiac surgical setting is the risk of thrombosis and thromboembolism. You reported no increase in risk of thrombosis or thromboembolism for those patients who received erythropoietin. Of note, in the United States the Food and Drug Administration has not approved erythropoietin for the specific use of decreasing red cell transfusion in cardiac surgery. So my question is, why do you think you did not find an increased risk for thrombosis? Is there some way you manage the patients differently, anticipating that this might be a risk for complications?

Dr Weltert. Good question. It was a surprise to us as well to find no complication at all. A few minor complications have been reported, such as rash, dizziness, fatigue, and a low incidence of thrombosis. So we expected to find some, approximately $1 \%$. As I said, we had absolutely no adverse event. 
One possible explanation, one possible pitfall of the study is that we ended the follow-up on day 4. So we can't ascertain if something has happened after discharge. I can tell you now confidentially that nothing has happened, because in Italy the patient usually comes back to you whatever happens, and nobody has come back.

Dr Koch. My final question pertains to cost. Another concern for the use of erythropoietin is the cost of administration. You were unable to demonstrate a cost benefit to the use of erythropoietin in comparison with reduced red cell transfusion. There are data to suggest that patients who received red cell transfusions have a longer time on the ventilator postoperatively, an increased infection rate that needs treatment, and prolonged intensive care unit and hospital length of stay. Can you speculate on whether or not if you included the cost of morbid events related to transfusion in your modeling, there would have been a cost benefit to the use of erythropoietin?

Dr Weltert. I know from performed second-level analysis that if we expanded the cost analysis to complications, this protocol would be convenient, but I had to stick to the simpler approach declared as the secondary end point. On further analysis, however, the protocol sort of moved patients 1 unit down. So if patients received 1 unit of blood, they were placed in a no transfusion category and so on. The protocol failed to demonstrate efficacy in the subset with more than 3 units of blood. There was no difference in terms of length of stay in hospital. So I can't say there is direct evidence of cost enhancements in this kind of analysis, but if we put everything together it will probably become convenient, yes.

Dr Koch. Thank you.

Dr Beat Walpoth (Geneva, Switzerland). At least experimentally there is some literature indicating that high-dose erythropoietin in a peri-ischemic model is brain and heart protective. Did you look at these issues in your large patient cohort?
Dr Weltert. I know these studies. When I planned the study, I tried to keep it as simple as possible with a clear primary end point and a simple secondary end point. The studies in literature usually fail to demonstrate statistically the evidence of association. I didn't want to get into that difficult topic, so I did not collect these data. However, the risks of thrombosis and ischemia are minimized by the inclusion criteria (patients with high hematocrit were excluded) and by the fact that every patient loses blood after the operation exactly on the day of maximal bone marrow output, thus lowering hematocrit and keeping it indirectly in a safe range.

Dr R. Duane Davis (Durham, NC). Can you comment on what you use for your antiplatelet therapy postoperatively, and how did you assess for myocardial ischemic or infarction events?

Dr Weltert. We followed the European guidelines for antiplatelet medications and diagnosis of ischemic events. More specifically, we used $100 \mathrm{mg}$ of acetyl salicylic acid since day 1 for the patient without previous ischemic cardiac events, and we used an association of aspirin and clopidogrel when myocardial infarction or ischemia occurred in the 15 days before surgery.

Dr Patricia M. Cruchley (Kitchener, Ontario, Canada). Your hemoglobin levels were quite high. Have you tried to separate out your patients in each group to see if the patients who were anemic received some benefit from the erythropoietin versus the whole group with their hemoglobin level of $134 \mathrm{~g} / \mathrm{dL}$ or whatever your average was?

Dr Weltert. Yes, absolutely. The subset of patients with a hemoglobin less than $9 \mathrm{~g} / \mathrm{dL}$ had the highest benefit from the protocol. The protocol does not give a statistical significant advantage on day 1 . We collected the analysis, but I didn't speak about that because there was no difference. But it shows a difference on day 4. The difference is inversely proportional to the starting point. So, yes, the subset who has the most benefit is the one starting with low hemoglobin. 